\title{
ON TORSION-FREE ABELIAN $k$-GROUPS
}

\author{
MANFRED DUGAS AND K. M. RANGASWAMY
}

\begin{abstract}
It is shown that a knice subgroup with cardinality $\aleph_{1}$, of a torsion-free completely decomposable abelian group, is again completely decomposable. Any torsion-free abelian $k$-group of cardinality $\aleph_{n}$ has balanced projective dimension $\leq n$.
\end{abstract}

Introduction. Recently, Hill and Megibben introduced the concept of a knice subgroup in their study of abelian $k$-groups [6] and also while considering the local Warfield groups in [5]. In this paper, we introduce a modified definition of a knice subgroup of a torsion-free abelian group. This helps us to extend the results of Hill and Megibben [6] and also simplify the proofs of their main theorems. Specifically we show that a knice subgroup with cardinality $\leq \aleph_{1}$, of a torsion-free completely decomposable abelian group, is again completely decomposable. This enables us to prove that any torsion-free abelian $k$-group (in particular, a separable group) of cardinality $\leq \aleph_{n}$ has balanced projective dimension $\leq n$.

All the groups that we consider here are torsion-free and abelian. We generally follow the notation and terminology of L. Fuchs $[\mathbf{3}]$. Let $P$ denote the set of all primes. By a height sequence we mean a sequence $s=\left(s_{p}\right), p \in P$, where each $s_{p}$ is a nonnegative integer or the symbol $\infty$. If $G$ is a torsion-free group and $x \in G$, then $|x|$ denotes the height sequence of $x$ where, for each $p \in P,|x|_{p}$ denotes the height of $x$ at the prime $p$. For any height sequence $s=\left(s_{p}\right), p s$ is the height sequence $\left(t_{p}\right)$, where $t_{p}=s_{p}+1$ and $t_{q}=s_{q}$ for all $q \neq p$. $G(s)$ denotes the subgroup $\{x \in G:|x| \geq s\} . G\left(s^{*}\right)$ is the subgroup generated by the set $\left\{x \in G(s): \sum_{p \in P}\left(|x|_{p}-s_{p}\right)\right.$ is unbounded $\}$. Two height sequences $\left(s_{p}\right)$ and $\left(t_{p}\right)$ are said to be equivalent if $\sum_{p \in P}\left|s_{p}-t_{p}\right|$ is finite.

Following Hill and Megibben [6], we write $G\left(s^{*}, p\right)$ for $G\left(s^{*}\right)+G(p s)$. If $a \in G$, then $\langle a\rangle_{*}$ denotes the pure subgroup generated by $a$.

DEFINITION [6]. Let $G$ be a torsion-free group.

(i) A subgroup $H$ of $G$ is said to be *-pure if $H$ is pure and, for all height sequences $s$ and primes $p, H \cap G\left(s^{*}, p\right)=H\left(s^{*}, p\right)$.

(ii) An element $x$ of $G$ is said to be primitive if for all height sequences $s$ equivalent to $|x|$ and for all primes $p$ for which $s_{p}=|x|_{p} \neq \infty, x \notin G\left(s^{*}, p\right)$. As pointed out in [6], $x$ is primitive if and only if $n x$ is primitive for any nonzero integer $n$. A useful remark is that if $x$ is primitive and $x \in G\left(s^{*}, p\right)$, then $x \in G(p s)$ or $\sum_{p \in P}\left(|x|_{p}-s_{p}\right)$ is unbounded.

As pointed out in [6], *-purity is transitive, inductive, and inherited by direct summands.

Received by the editors September 24, 1985 and, in revised form, January 1986.

1980 Mathematics Subject Classification (1985 Revision). Primary 20K20; Secondary 20K27. 
Proposition 1. $y \in G$ is primitive if and only if the pure subgroup $\langle y\rangle_{*}$ generated by $y$ is *-pure.

PROOF. Let $y$ be primitive. In view of the preceding remarks, every nonzero element of $\langle y\rangle_{*}=H$ is primitive. Thus if $a \in H \cap G\left(s^{*}, p\right)$, then by the primitivity of $a$, either $a \in H \cap G(p s)=H(p s)$ or $\sum\left(|a|_{p}-s_{p}\right)$ is unbounded. Hence $a \in H(p s)$ or $a \in H\left(s^{*}\right)$. Thus $H$ is *-pure. Conversely, suppose $H=\langle y\rangle_{*}$ is *-pure, where we can choose $|y|=s=$ the type of $H$. Clearly $H\left(s^{*}\right)=0$. Since $y \notin H(p s)$, $y \notin H\left(s^{*}, p\right)=H \cap G\left(s^{*}, p\right)$ for all primes $p$ for which $H$ is not $p$-divisible. So $y$ must be primitive.

The following modification of a result of Hill and Megibben is crucial in later discussions.

PROPOSITION 2. If $G$ is separable, then any finite rank *-pure subgroup $H$ is a completely decomposable summand.

ProOF. The proof is by induction on the rank of $H$. Let $H$ have rank one, say, $H=\langle y\rangle_{*}$. Since $H$ is contained in a completely decomposable summand of $G$, we may assume $G=\left\langle x_{1}\right\rangle * \oplus \cdots \oplus\left\langle x_{n}\right\rangle *$ and $y=x_{1}+\cdots+x_{n}$. Clearly $|y| \leq\left|x_{i}\right|$, $i=1, \ldots, n$. Since, by Proposition $1, y$ is primitive, at least one of the $x_{i}$ 's must have the same type $s$ as $y$ (as $y \notin G\left(s^{*}\right)$ ). Rearranging the $x_{i}$, if necessary, write $y=y^{\prime}+z$, where $y^{\prime}=x_{1}+\cdots+x_{k}$ with $x_{1}, \ldots, x_{k}$ having the same type as $y$ and $z=x_{k+1}+\cdots+x_{n}$. Then $\left\langle y^{\prime}\right\rangle_{*}$, being pure, is a summand of the homogeneous completely decomposable group $\left\langle x_{1}\right\rangle * \oplus \cdots \oplus\left\langle x_{k}\right\rangle *$ and hence a summand of $G$, say $G=\left\langle y^{\prime}\right\rangle_{*} \oplus N$ with $z \in N$. Then $G=\langle y\rangle_{*} \oplus N$.

Suppose $H$ has finite rank $n>1$ and that the result holds for $*$-pure subgroups of smaller rank. Let $y \in H$ be an element of maximal type $s$. We claim that $y$ is primitive. Since this happens if $n y$ is primitive, we may assume without loss of generality that $|y|=s$. Then $H\left(s^{*}\right)=0$ and for each prime $p$ for which $s_{p}=|y|_{p} \neq$ $\infty, y \notin H(p s)=H\left(s^{*}, p\right)=H \cap G\left(s^{*}, p\right)$. Thus $y$ is primitive in $G$. Then we can write $G=\langle y\rangle * \oplus K$ and $h=\langle y\rangle * \oplus L$, where $L=H \cap K$ is $*$-pure of rank $n-1$ and hence a completely decomposable summand of $G$ contained in $K$. This proves the result.

As a corollary we obtain a simple 'natural' proof of the classical theorem of Fuchs on summands of separable groups.

Corollary 3. If $G$ is separable and $G=A \oplus B$, then $A$ is separable.

Proof. Let $p_{A}: G \rightarrow A, p_{B}: G \rightarrow B$ be coordinate projections. Let $X$ be a finite subset of $A$. Then $X \subseteq G_{1}$, a finite rank completely decomposable summand of $G$. Now there is a finite rank completely decomposable summand $G_{2}$ of $G$ such that

$$
G_{1} \subseteq p_{A}\left(G_{1}\right)+p_{B}\left(G_{1}\right) \subseteq G_{2} .
$$

Proceeding like this we get an increasing chain of subgroups

$$
G_{1} \subseteq p_{A}\left(G_{1}\right)+p_{B}\left(G_{1}\right) \subseteq G_{2} \subseteq p_{A}\left(G_{2}\right)+p_{B}\left(G_{2}\right) \subseteq \cdots
$$

If

$$
G^{*}=\bigcup_{n=1,2, \ldots} G_{n}, \quad A^{*}=\bigcup_{n=1,2, \ldots} p_{A}\left(G_{n}\right), \quad \text { and } \quad B^{*}=\bigcup_{n=1,2, \ldots} p_{B}\left(G_{n}\right)
$$


then $G^{*}=A^{*} \oplus B^{*}$. Since $G^{*}$ is *-pure completely decomposable, so is $A^{*}$. Let $X \subseteq D$, a finite rank summand of $A^{*}$. Then $D$ is *-pure in $G$. By the separability of $G$ and Proposition 2, $D$ is a summand of $G$. This proves that $A$ is separable.

We now give a modified definition of a knice subgroup of a torsion-free group.

DEFINITION. (i) A subgroup $N$ of a torsion-free group $G$ is said to be knice if for each finite subset $X$ of $G$, there is a finite rank completely decomposable subgroup $H$ such that $N+H=N \oplus H$ is *-pure and $X \subseteq N \oplus H$.

(ii) A group $G$ is called a $k$-group, if $\{0\}$ is knice.

It is clear that a knice subgroup is always balanced. Conversely, as pointed out in [6], a balanced subgroup $B$ is knice in $G$ if and only if $G / B$ is a $k$-group.

REMARK. Our definition of knice is slightly different from that of Hill and Megibben [5, 6]. For torsion-free groups it is readily seen that "knice" is our sense is the same as "pure knice" in the sence of Hill and Megibben [6].

We begin with a simple characterization of $K$-groups.

Proposition 4. (i) $G$ is a k-group if and only if $G=C / B$, where $C$ is completely decomposable and $B$ is knice in $C$.

(ii) $G$ is a $k$-group if and only if every countable subset of $G$ can be embedded in $a$ *-pure completely decomposable subgroup of $G$.

PrOOF. (i) Let $G$ be a $K$-group. As with any torsion-free group we can write $G=C / B$, where $C$ is completely decomposable and $B$ balanced $[3$, p. 117, Exercise 16]. The rest follows from Theorem 4.3 in [6].

(ii) follows since the union of an increasing chain of finite rank *-pure completely decomposable subgroups is, by Proposition 2, again completely decomposable and *-pure.

COROLlaRY 5. A countable knice subgroup of a completely decomposable group is a summand.

Using our modified definition of knice subgroups we give below a much simpler and direct proof of Theorem 4.8 of Hill and Megibben [6].

THEOREM 6 [6]. If $G$ is a $K$-group and $H$ is knice in $G$, then $H$ is a k-group.

Proof. Let $X$ be a finite subset of $H$. Since $G$ is a $k$-group, there is a finite rank *-pure completely decomposable subgroup $A_{1}$ of $G$ containing $X$. Since $H$ is knice, $A_{1} \subseteq H \oplus M_{1}$, where $M_{1}$ is finite rank completely decomposable and $H \oplus M_{1}$ is *-pure. Actually $A_{1} \subseteq H_{1} \oplus M_{1}$, where $H_{1}=f\left(A_{1}\right)$, $f$ being the coordinate projection $H \oplus M_{1} \rightarrow H$. Since $H_{1} \oplus M_{1}$ has finite rank and $G$ is a $k$-group, $H_{1} \oplus M_{1} \subseteq A_{2}$, a finite rank *-pure completely decomposable subgroup of $G$. Since $H$ is knice,

$$
A_{2} \subseteq H_{2} \oplus M_{2} \subseteq H \oplus M_{2},
$$

where $H \oplus M_{2}$ is *-pure, $H_{2}=f\left(A_{2}\right), f: H \oplus M_{2} \rightarrow H$ being the projection map and $M_{2}$ is finite rank completely decomposable. Here $M_{2}$ can be chosen to contain $M_{1}$ : Indeed, if

$$
L=H \oplus M_{1} \subseteq H \oplus M_{2},
$$

then $L=H \oplus\left(L \cap M_{2}\right)$ by modular law. Since $L$ is *-pure in $G$, so is $L \cap M_{2}$ and Proposition 2 implies that $L \cap M_{2}$ is a summand of $M_{2}$, say, $M_{2}=\left(L \cap M_{2}\right) \oplus M_{2}^{\prime}$. Then

$$
H \oplus M_{2}=H \oplus\left(L \cap M_{2}\right) \oplus M_{2}^{\prime}=H \oplus M_{1} \oplus M_{2}^{\prime} .
$$


Hence we can replace $M_{2}$ by $M_{1} \oplus M_{1}^{\prime}$ and assume that $M_{1} \subseteq M_{2}$. Then $H_{2}=$ $f\left(A_{2}\right) \supseteq f\left(A_{1}\right)=H_{1}$. Proceeding like this, we get the following increasing chains of subgroups of $G$ :

$$
\begin{aligned}
& A_{1} \subseteq H_{1} \oplus M_{1} \subseteq A_{2} \subseteq H_{2} \oplus M_{2} \subseteq \cdots \\
& M_{2} \subseteq M_{2} \subseteq \cdots, \quad \text { and } \quad H_{1} \subseteq H_{2} \subseteq \cdots
\end{aligned}
$$

If

$$
A^{*}=\bigcup_{n=1,2, \ldots} A_{n}, \quad M^{*}=\bigcup_{n=1,2, \ldots} M_{n}, \quad \text { and } H^{*}=\bigcup_{n=1,2, \ldots} H_{n},
$$

then $A^{*}=M^{*} \oplus H^{*}$. Since, for each $n, A_{n}$ is *-pure completely decomposable of finite rank, $A_{n}$ is a summand of $A_{n+1}$ by Proposition 2 and so $A^{*}$ is *-pure completely decomposable. Hence its summand $H^{*}$ is a $*$-pure completely decomposable subgroup of $H$ containing $X$. Thus $H$ is a $k$-group.

A modification of the above proof readily shows that a knice subgroup of a separable group is again separable. A natural question is about knice subgroups of completely decomposable groups. Should they necessarily be completely decomposable? Theorems 7 and 9 below consider this question.

THEOREM 7. A knice subgroup $B$ of a completely decomposable group $C$ is $\aleph_{1}$-separable.

ProOF. Let $A=C / B$. By Proposition 4(i), $A$ is a $k$-group. Let $Y=$ $\left\{y_{1}, y_{2}, \ldots\right\}$ be a countable subset of $B$. Write $C=\bigoplus_{i \in I} X_{i}$, where the $X_{i}$ are rank one groups. Let $y_{1} \in C_{1}$ a direct sum of finitely many $X_{i}$ 's. Since $C / B$ is a $k$-group, $\left(C_{1}+B\right) / B \subset A_{1} / B$, a $*$-pure finite rank completely decomposable subgroup of $C / B$. Since $B$ is balanced, $A_{1}=B \oplus S_{1}, S_{1} \cong A_{1} / B$. Let $p: B \oplus S_{1} \rightarrow B$ be the coordinate projection. Then $C_{1} \subseteq B_{1} \oplus S_{1}$, where $B_{1}=p\left(C_{1}\right)$. Let $C_{2}$ be a direct sum of finitely many $X_{i}$ 's such that $\left\{y_{2}\right\} \cup\left(B_{1} \oplus S_{1}\right) \subseteq C_{2}$. Again since $C / B$ is a $k$-group, $\left(C_{2}+B\right) / B \subseteq A_{2} / B$, a *-pure completely decomposable finite rank subgroup of $C / B$. By Proposition $2, A_{2} / B=\left(A_{1} / B\right) \oplus \bar{S}$. Since, in addition, $B$ is balanced, we can write $A_{2}=B \oplus S_{2}$ with $S_{2} \cong A_{2} / B$ and $S_{1} \subseteq S_{2}$. Proceeding like this we get the following increasing chains:

$$
\begin{aligned}
& C_{1} \subseteq B_{1} \oplus S_{1} \subseteq C_{2} \subseteq B_{2} \oplus S_{2} \subseteq \cdots, \\
& S_{1} \subseteq S_{2} \subseteq \cdots, \quad \text { and } \quad B_{1} \subseteq B_{2} \subseteq \cdots .
\end{aligned}
$$

If

$$
C^{*}=\bigcup_{n-1,2, \ldots} C_{n}, \quad B^{*}=\bigcup_{n=1,2, \ldots} B_{n}, \quad \text { and } \quad S^{*}=\bigcup_{n=1,2, \ldots} S_{n},
$$

then $C^{*}=B^{*} \oplus S^{*}$. Since $C^{*}$ is a summand of $C, B^{*}$ is a completely decomposable summand (of $C$ and) of $B$ containing $Y$. Hence $B$ is $\aleph_{1}$-separable.

Proposition 8. Each k-group $G$ of cardinality $m>\aleph_{0}$ is the union of a smooth chain of $k$-groups $\left\{K_{\alpha}\right\}$, where each $K_{\alpha}$ has the cardinality less than $m$ and is *-pure in $G$.

PROOF. Let $\left\{x_{\alpha}: \alpha<\tau\right\}$ be a well-ordering of the elements of $G$. We construct the $K_{\alpha}$ inductively. Let $\beta<\alpha$. Suppose $K_{\alpha}$ have been constructed for all $\alpha<\beta$, so that $x_{\gamma} \in K_{\gamma+1}$ for all $\gamma<\alpha$, each $K_{\alpha}$ is a $k$-group, and is *-pure in $G$. If $\beta$ is a limit ordinal, define $K_{\beta}=\bigcup_{\alpha<\beta} K_{\alpha}$. Suppose $\beta=\alpha+1$. Let $K_{\alpha, 1}=\left\langle x_{\alpha}\right\rangle+\sum S$, 
where $S$ runs over all the *-pure finite rank completely decomposable subgroups of $K$. Let $K_{\alpha, 2}=\sum S$, where $S$ runs over all the *-pure finite rank completely decomposable subgroups of $G$ containing finite subsets of $K_{\alpha, 1}$, and so on. Define $K_{\beta}=\bigcup_{n=1,2, \ldots} K_{\alpha, n}$. Clearly $K_{\beta}$ is a $k$-group of cardinality $<m$. It is readily seen that $K_{\beta}$ is *-pure in $G$.

$\mathrm{Ou}$ next theorem considers the knice subgroups of completely decomposable groups and extends Theorem 5.4 of [6].

THEOREM 9. If $G$ is completely decomposable, then any knice subgroup $H$ of cardinality $\leq \aleph_{1}$, is completely decomposable.

We first wish to review the notion of compatibility introduced by Hill $[\mathbf{4}]$ and a terminology connected with Hill's third axiom of countability.

Two subgroups $A$ and $B$ of $G$ are said to be compatible, in symbols, $A \| B$, if the following condition holds: If $(a, b) \in A \times B$ and if $s$ is a height sequence with $s \leq|a+b|$, then there exists an element $b^{\prime} \in A \cap B$ such that $s \leq\left|a+b^{\prime}\right|$. The proof of Lemma 1 of Hill [4] implies that if $H$ is a balanced subgroup of $G$ and if $S$ is a subset of $G$, then there is a subgroup $B$ of $G$ such that $S \subset B, B \| H$, and $|B|=|S|$ if $S$ is infinite. We shall also use the following remark pointed out in [6]. (in the proof of Theorem 5.4): If $H$ is balanced in $G$ and $A$ is a pure subgroup of $G$ with $A \| H$, then $A \cap H$ is a balanced subgroup of $A$. In order to prove Theorem 9 , we need the following important lemma:

LEMMA 10. Suppose $G$ is completely decomposable of regular cardinality $\kappa, H$ is a knice subgroup of $G, G=\bigcup_{\alpha<\kappa} G_{\alpha}$, and $G / H=\bigcup_{\alpha<\kappa} D_{\alpha}$ are smooth filtrations, where $G_{\alpha}$ are summands of $G$ with $\left|G_{\alpha}\right|<\kappa$ and $D_{\alpha}$ are $k$-groups of $\left|D_{\alpha}\right|<\kappa$. Then there is a closed and unbounded subset $C$ of $\kappa$ such that $H=\bigcup_{\alpha \in C} H_{\alpha}$, where $H_{\alpha}=H \cap G_{\alpha}$ is knice in $H$ for each $\alpha \in C$, and $\left(G_{\alpha}+H\right) / H=D_{\alpha}$.

ProOF. Now $\bigcup_{\alpha<\kappa} D_{\alpha}=G / H=\bigcup_{\alpha<\kappa}\left(G_{\alpha}+H\right) / H$ are two $\kappa$-filtrations of $G / H$, so by a standard argument (cf. [2, p. 26]), $C=\left\{\alpha<\kappa \mid D_{\alpha}=\left(G_{\alpha}+H\right) / H\right\}$ is a closed and unbounded set. Let $H_{\alpha}=H \cap G_{\alpha}$ for all $\alpha \in C$. We claim that $D=\left\{\alpha \in C \mid G_{\alpha} \| H\right\}$ is also closed and unbounded. Since the union of an increasing chain of subgroups $S$ each compatible to $H$ is again compatible to $H$, $D$ is closed. We wish to show that $D$ is unbounded in $C$. Let $\alpha \in C$ so that $\left(G_{\alpha}+H\right) / H=D_{\alpha}$. Since $H$ is balanced, by Lemma 1 of Hill [4], $G_{\alpha} \subseteq A_{1}$, where $A_{1} \| H$ and $\left|A_{1}\right|=\left|G_{\alpha}\right|$. Since $\left\{G_{\alpha}\right\}$ is a $\kappa$-filtration of $G$, there is $\alpha<\alpha_{1} \in C$ such that $A_{1} \subseteq G_{\alpha_{1}}$. By Lemma 1 of Hill [4], there exists $A_{2} \| H$ with $G_{\alpha_{1}} \subseteq A_{2}$ and $\left|A_{2}\right|=\left|G_{\alpha_{1}}\right|$. Proceeding like this we get an increasing chain

$$
G_{\alpha} \subseteq A_{1} \subseteq G_{\alpha_{1}} \subseteq A_{2} \subseteq G_{\alpha_{2}} \subseteq \cdots, \quad \text { where } \alpha_{i} \in C .
$$

Then $\bigcup_{n<\omega} G_{\alpha_{n}}=\bigcup_{n<\omega} A_{n}=G_{\beta}$, where $\beta=\lim \alpha_{n}$, is compatible to $H$ and $\beta \in C$, since $C$ is closed. Hence $\beta \in D$ and clearly $\alpha \leq \beta$. Thus $D$ is unbounded. Now for $\alpha \in D, G_{\alpha} / H_{\alpha} \cong D_{\alpha}$ is a $k$-group, $H$ is balanced and $G_{\alpha} \| H$. This implies that $H_{\alpha}$ is balanced in $G_{\alpha}, G_{\alpha} / H_{\alpha}$ is a $k$-group, and so $H_{\alpha}$ is knice in $G_{\alpha}$ and hence in $G$ by the transitivity of kniceness (Proposition 4.10(i), [6]). Since $H$ is knice in $G, H_{\alpha}$ is a knice subgroup of $H$, by Proposition 4.10(iv) of [6].

PROOF OF THEOREM 9. Since $G$ is completely decomposable we can assume $|G| \leq \aleph_{1}$. As in Lemma 10 , choose a smooth filtration $\left\{G_{\alpha}\right\}$ for $G$ and $\left\{D_{\alpha}\right\}$ for $G / H$ with stated properties. Proposition 8 guarantees the existence of the filtration 
$\left\{D_{\alpha}\right\}$. As before let $H_{\alpha}=H \cap G_{\alpha}$. Since $|G / H| \leq \aleph_{1}$, each $D_{\alpha}$ will be a countable $k$-group and hence completely decomposable. Now $H_{\alpha+1} / H_{\alpha}$ is countable, and $H_{\alpha}$ knice in $H_{\alpha+1}$ implies $H_{\alpha+1} / H_{\alpha}$ is a countable $k$-group and hence completely decomposable so that $H_{\alpha+1}=H_{\alpha} \oplus K_{\alpha}$. Then $H=\bigcup H_{\alpha}=\bigoplus K_{\alpha}$ is completely decomposable.

COROLLARY 11. A k-group of cardinality $\aleph_{1}$ has balanced projective dimension $\leq 1$.

THEOREM 12. Any k-group (in particular, a separable group) $G$ of cardinality $\leq \aleph_{n}$ has balanced projective dimension $\leq n$.

Proof. By Corollary 11, the theorem holds if $n=1$. Suppose the theorem is true if $1 \leq n<m$. Let $|G|=\aleph_{m}$. By Proposition 4(i), $G=C / H$, where $C$ completely decomposable and $H$ a knice subgroup of $C$. By Lemma $8, G=$ $\bigcup_{\alpha<\aleph_{n}} D_{\alpha}$ is a smooth filtration of *-pure $k$-groups $D_{\alpha}$ with $\left|D_{\alpha}\right| \leq \aleph_{m-1}$. By Lemma 10, this induces a smooth filtration $H=\bigcup_{\alpha \in C} H_{\alpha}$ of knice subgroups $H_{\alpha}$ of $H$, where $C$ is a cub of $\aleph_{m}$. Then for each $\alpha, H_{\alpha+1} / H_{\alpha}$ is a $k$-group of cardinality $\leq \aleph_{m-1}$ and so has balanced projective dimension $\leq m-1$.

Note that each $H_{\alpha}$ is in particular balanced in $H$. Then, by Auslander's Theorem $[\mathbf{1}], H$ has balanced projective dimension $\leq m-1$. This implies that $G$ has balanced projective dimension $\leq m$.

We would like to thank D. Arnold, W. Wickless, and the referee for their helpful comments, and, in particular, for pointing out the incorrect argument in the proof of Lemma 10.

\section{REFERENCES}

1. H. Auslander, On the dimension of modules and algebras. III, Nagoya Math. J. 9 (1955), 67-77.

2. P. Eklof, Set theoretic methods in homological algebra and abelian groups, Presses Univ. Montreal, Montreal, 1980.

3. L. Fuchs, Infinite abelian groups, vol. II, Academic Press, New York, 1973.

4. P. Hill, Isotype subgroups of totally projective groups, Lecture Notes in Math., vol. 874, SpringerVerlag, Berlin and New York, 1981, pp. 305-321.

5. P. Hill and C. Megibben, Axiom 3 modules, Trans. Amer. Math. Soc. 295 (1986), 715-734.

6. __ Torsion-free groups, Trans. Amer. Math. Soc. 295 (1986), 735-751.

Department of Mathematics, University of Colorado, Colorado Springs, COLORADO 80933-7150 